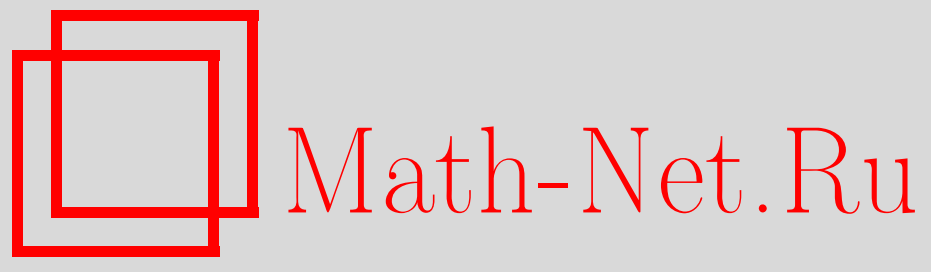

И. В. Вьюгин, Проблема Римана-Гильберта для скалярных фуксовых уравнений и родственные задачи, УМH, 2011, том 66, выпуск 1, 37-64

DOI: https://doi.org/10.4213/rm9405

Использование Общероссийского математического портала Math-Net.Ru подразумевает, что вы прочитали и согласны с пользовательским соглашением http://www . mathnet.ru/rus/agreement

Параметры загрузки:

IP: 54.198 .55 .26

26 апреля 2023 г., 10:12:28

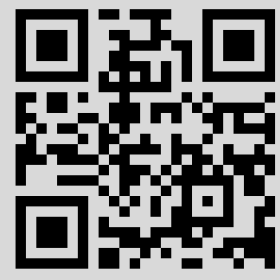




\title{
Проблема Римана-Гильберта для скалярных фуксовых уравнений и родственные задачи
}

\author{
И. В. Вьюгин
}

\begin{abstract}
Работа посвящена проблеме Римана-Гильберта для скалярных фуксовых уравнений: задаче построения скалярного фуксова уравнения по представлению монодромии и набору особых точек. Основную часть работы представляют результаты А.А. Болибруха [5], М. Ван-дер-Пута и М. Зингера [7] и автора [10], обобщенные в единую теорему, снабженную новым доказательством. Обсуждаются также некоторые из возможных приложений этих результатов.
\end{abstract}

Библиография: 16 названий.

Ключевые слова: фуксовы уравнения и системы, монодромия, проблема Римана-Гильберта, расслоение, связность.

\section{СОДЕРЖАНИЕ}

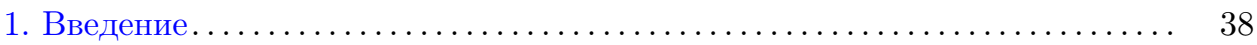

2. Основные понятия и формулировки ...................... 39

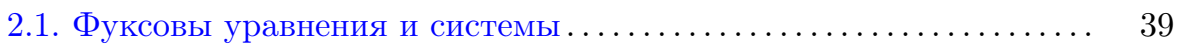

2.2. Формулировки основных результатов .................. 40

2.3. Локальная структура фундаментальной системы решений..... 42

2.4. Векторные расслоения с логарифмическими связностями над

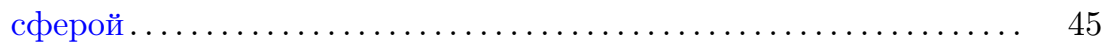

2.5. Глобальная классификация голоморфных расслоений над $\overline{\mathbb{C}} \ldots . .47$

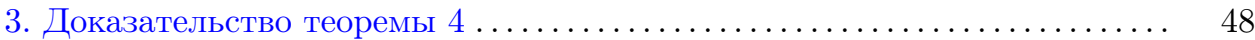

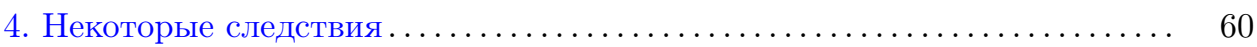

4.1. Контрпримеры к проблеме Римана-Гильберта для скалярных

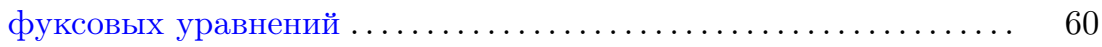

4.2. Недеформируемость фуксовых уравнений .............. 61

Список литературы .................................. 63

Работа выполнена при поддержке РФФИ (грант № 11-01-00384_а) и программы “Ведущие научные школы" (грант НШ-8508.2010.1). 


\section{1. Введение}

История проблемы Римана-Гильберта началась в 1857 г., когда Б. Риман рассмотрел вопрос о восстановлении фуксова уравнения по его особым точкам и монодромии (см. [1]). На Парижском международном математическом конгрессе 1900 г. Д. Гильберт включил эту задачу в список знаменитых проблем под номером 21, сформулировав ее следующим образом (см. [2]):

показать, что всегда существует линейное дифберенциальное уравнение фуксова типа с заданными особыми точками и заданной группой монодромии.

В качестве мотивировки этой задачи он лишь отмечает, что решение данной проблемы придало бы законченный вид аналитической теории линейных дифференциальных уравнений.

Под проблемой Римана-Гильберта обычно понимаются несколько различных задач. Центральным вопросом этой работы будет проблема Римана-Гильберта для скалярных фуксовых уравнений. Общий отрицательный ответ в этой задаче был известен еще А. Пуанкаре, который вычислил размерности (число свободных параметров) множеств всех представлений и всех скалярных фуксовых уравнений и показал, что, за исключением случая, к которому относится уравнение Гаусса, размерность множества скалярных фуксовых уравнений меньше размерности множества всех представлений. Несмотря на то что почти для всех представлений и наборов особых точек этот вариант проблемы Римана-Гильберта имеет отрицательное решение, очень трудно найти условия положительной или отрицательной разрешимости этой задачи. Практически первым источником явных контрпримеров к этой задаче стала теорема 3 , доказанная А. А. Болибрухом только в 1994 г. и утверждающая, что контрпримеры к проблеме Римана-Гильберта для фуксовых систем являются контрпримерами и для этой задачи.

В работе представлены основные результаты по данной проблеме, известные к настоящему моменту. В первую очередь это результаты, связывающие три варианта проблемы Римана-Гильберта. Показано, что проблема Римана-Гильберта для скалярных фуксовых уравнений в точности эквивалентна некоторому варианту проблемы Римана-Гильберта для фуксовых систем и некоторому варианту задачи построения логарифмической связности в векторном расслоении заданного голоморфного типа над сферой. В качестве следствия из этих результатов получены новые серии контрпримеров к проблеме Римана-Гильберта для скалярных фуксовых уравнений. Это первые явные большие серии таких примеров. Также доказано, что не бывает представлений с не менее чем четырьмя образующими, которые могут быть реализованы как представление монодромии скалярных фуксовых уравнений с любым набором особых точек, точнее, доказано, что множество таких наборов особых точек всегда имеет положительную коразмерность.

В заключение введения отметим, что решения некоторых известных нелинейных уравнений, таких как шестое уравнение Пенлеве или системы Гарнье, выражаются через коэффициенты изомонодромных семейств фуксовых уравнений, имеющих дополнительные “ложные" (где решения не ветвятся) особые 
точки. Количество таких точек дает теорема 1. Изучение условий положительной разрешимости проблемы Римана-Гильберта в контексте этой задачи означает описание положения подвижных особенностей вышеперечисленных уравнений.

\section{2. Основные понятия и формулировки}

2.1. Фуксовы уравнения и системы. Рассмотрим линейное дифференциальное уравнение

$$
y^{(p)}+b_{1}(z) y^{(p-1)}+\cdots+b_{p}(z) y=0, \quad z \in \mathbb{C},
$$

с рациональными на комплексной плоскости коэффициентами $b_{1}(z), \ldots, b_{p}(z)$, голоморфными вне множества особых точек $a_{1}, \ldots, a_{n}$.

Представлением монодромии или монодромией данного уравнения называется представление

$$
\chi: \pi_{1}\left(\overline{\mathbb{C}} \backslash\left\{a_{1}, \ldots, a_{n}\right\}, z_{0}\right) \longrightarrow \mathrm{GL}(p, \mathbb{C})
$$

фундаментальной группы пространства $\overline{\mathbb{C}} \backslash\left\{a_{1}, \ldots, a_{n}\right\}$ в группу невырожденных комплексных матриц порядка $p$, которое определяется следующим образом. В окрестности неособой точки $z_{0}$ возьмем базис $\left(y_{1}(z), \ldots, y_{p}(z)\right)$ пространства решений уравнения (1). При аналитическом продолжении функций $y_{1}(z), \ldots, y_{p}(z)$ вдоль произвольной петли $\gamma$, исходящей из точки $z_{0}$ и лежащей в $\overline{\mathbb{C}} \backslash\left\{a_{1}, \ldots, a_{n}\right\}$, базис $\left(y_{1}, \ldots, y_{p}\right)$ переходит в (вообще говоря, другой) базис $\left(\tilde{y}_{1}, \ldots, \tilde{y}_{p}\right)$. Два базиса связаны с помощью невырожденной матрицы перехода $G_{\gamma}$, соответствующей петле $\gamma$ :

$$
\left(y_{1}, \ldots, y_{p}\right)=\left(\tilde{y}_{1}, \ldots, \tilde{y}_{p}\right) G_{\gamma} .
$$

Отображение $[\gamma] \mapsto G_{\gamma}$ (которое зависит только от гомотопического класса $[\gamma]$ петли $\gamma$ ) и задает представление $\chi$. Матрицей монодромии уравнения (1) в особой точке $a_{i}$ (относительно базиса $\left(y_{1}, \ldots, y_{p}\right)$ ) называется матрица $G_{i}$, соответствующая простой петле $\gamma_{i}$, обходящей точку $a_{i}$, т. е. $G_{i}=\chi\left(\left[\gamma_{i}\right]\right)$.

Особая точка $a_{i}$ уравнения (1) называется фуксовой, если коэффициент $b_{j}(z)$ имеет в этой точке полюс порядка не более $j, j=1, \ldots, p$. Согласно теореме Фукса (см. [3]), особая точка $a_{i}$ является фуксовой тогда и только тогда, когда она регулярна (т.е. когда любое решение имеет не более чем степенной рост в окрестности точки $a_{i}$ ). Уравнение (1) называется фуксовым, если все его особые точки фуксовы.

Задача о построении фуксова уравнения (1) с заданными особыми точками $a_{1}, \ldots, a_{n}$ и заданным представлением монодромии (2) в общем случае имеет отрицательное решение, поскольку число параметров, от которых зависит такое уравнение, меньше числа параметров, от которых зависит множество представлений $\chi$ (см. [3], а также следующий п. 2.2).

Наряду с уравнением (1) можно рассмотреть линейную систему

$$
\frac{d y}{d z}=B(z) y, \quad y(z) \in \mathbb{C}^{p}, \quad B(z) \in \operatorname{Mat}_{p \times p}(\mathbb{C}),
$$


из $p$ уравнений с мероморфной на сфере Римана матрицей $B(z)$, голоморфной вне точек $a_{1}, \ldots, a_{n}$. Представление монодромии данной системы определяется так же, как и для уравнения (1), нужно только вместо строки $\left(y_{1}, \ldots, y_{p}\right)$ рассмотреть фундаментальную матрицу $Y(z)$ - матрицу, столбцы которой образуют базис в пространстве решений системы. Представление монодромии линейной системы имеет такой же вид (2).

Особая точка $a_{i}$ системы (3) называется фуксовой, если матрица $B(z)$ имеет простой полюс (полюс первого порядка) в этой точке. Фуксова особая точка линейной системы всегда является регулярной, хотя регулярная особенность не обязана быть фуксовой (см. [3]). Система (3) называется фуксовой, если все ее особые точки фуксовы. Нетрудно показать, что фуксова на сфере Римана система представляется в следующем виде:

$$
\frac{d y}{d z}=\left(\sum_{i=1}^{n} \frac{B_{i}}{z-a_{i}}\right) y
$$

причем отсутствие особенности в точке $z=\infty$ эквивалентно условию $\sum_{i=1}^{n} B_{i}=0$.

2.2. Формулировки основных результатов. Главным предметом исследования данной работы будет задача построения скалярного фуксова уравнения (1) по его представлению монодромии (2), называемая проблемой РиманаГилъберта для скалярных фуксовых уравнений. Еще А. Пуанкаре вычислил размерность (число определяющих комплексных параметров) множества всех скалярных фуксовых уравнений, а также множеств всех представлений и всех фуксовых систем, имеющих заданную размерность $p$ и число особых точек $n$. Нетрудно показать (см. [3]), что эти размерности равны

$$
N_{\text {eq }}=\frac{(n-2) p(p+1)}{2}+p, \quad N_{\text {repr }}=N_{\text {syst }}=(n-2) p^{2}+1,
$$

где $N_{\text {eq }}, N_{\text {repr }}$ и $N_{\text {syst }}$ - размерности множеств уравнений, представлений и систем соответственно, рассматриваемых с точностью до эквивалентности. Получается, что

$$
N_{\text {eq }}<N_{\text {repr }}
$$

всегда кроме случая $n=3, p=2$, к которому относится гипергеометрическое уравнение. Отсюда сразу следует, что положительное решение проблемы Римана-Гильберта для скалярных фуксовых уравнений при $(n, p) \neq(3,2)$ возможно только в исключительных случаях, имеющих положительную коразмерность в пространстве представлений (2). Этого нельзя сказать о классической проблеме Римана-Гильберта для фуксовых систем, где размерности множеств совпадают, $N_{\text {syst }}=N_{\text {repr }}$, и проблема Римана-Гильберта имеет положительное решение для большинства представлений (2). Отметим, что первый пример представления (2), которое не может быть реализовано в качестве представления монодромии фуксовой системы, был построен А. А. Болибрухом только в 1989 г. (см. [3], [4]).

Для того чтобы построить уравнение (1) по монодромии (2), как правило, нужно вводить дополнительные (“ложные”) особенности $b_{1}, \ldots, b_{m}$, в которых 
решения не ветвятся. В случае, когда монодромия неприводима, минимальное количество таких особых точек может быть задано формулой из следующей теоремы.

Теорема 1 (А. А. Болибрух [5]). Минимально возможное число $m_{0}$ дополнительных ложных особых точек скалярного фуксова уравнения (1) с заданной неприводимой монодромией (2) задается следующей формулой:

$$
m_{0}=\frac{(n-2) p(p-1)}{2}-\gamma_{\max }(\chi) \geqslant \frac{(n-2) p(p-1)}{2}-p+1,
$$

где $\gamma_{\max }(\chi)$ - максимальный фуксов вес представления $\chi$ (см. определение в $n$. 2.5).

СлЕДСТВИЕ 1. При условии, что существует расслоение с логарифмической связностъю, имеющей неприводимое представление монодромии (2), с особъми точками $a_{1}, \ldots, a_{n}$ и типом расщепления

$$
K=(0,(n-2), \ldots,(n-2)(p-1)),
$$

представление (2) может быть реализовано в качестве монодромии фуксова уравнения (1) без дополнителъных особенностей.

Это следствие сразу получается из теоремы 1 и определения максимального фуксова веса представления. Для случая приводимого представления существует оценка необходимого количества дополнительных особенностей.

Теорема 2 (И. В. Вьюгин, Р.Р. Гонцов [6]). Для произвольного представления (2) существует скалярное фуксово уравнение (1) с данной монодромией, число $m$ дополнительных ложных особых точек которого удовлетворяет неравенству

$$
m \leqslant \frac{(n+1) p(p-1)}{2}+1 .
$$

Tеорема 3 (А. А. Болибрух [5]). По любому фуксову уравнению (1) на сфере Римана можно построить фуксову систем

$$
\frac{d y}{d z}=\left(\sum_{i=1}^{n} \frac{B_{i}}{z-a_{i}}\right) y
$$

с теми же особыми точками и монодромией.

Эта теорема была передоказана методами дифференциальной алгебры в книге М. Ван-дер-Пута и М. Зингера [7], где было также показано, что фуксову систему можно выбрать имеющей почти треугольную форму. Упрощенное доказательство теоремы 3 опубликовано в [8] и перепечатано в качестве приложения 1 в [3]. В работе [9] эта теорема обобщена на случай уравнений и систем с иррегулярными особыми точками, т. е. произвольных уравнений с рациональными коэффициентами.

Основным результатом настоящей работы является следующая теорема 4, анонсированная в [10], которая частично усиливает и обобщает приведенные выше результаты. 
Теорема 4. Следующие три задачи эквивалентны.

I. Построить по представлению х и набору точек $a_{1}, \ldots, a_{n}$ буксово уравнение

$$
\frac{d^{p} y}{d z^{p}}+b_{1}(z) \frac{d^{p-1} y}{d z^{p-1}}+\cdots+b_{p}(z) y=0
$$

имеющее представление монодромии ұ и заданный набор особых точек.

II. Построить по представлению $\chi$ и набору точек $a_{1}=0, a_{2}, \ldots, a_{n-1}$, $a_{n}=\infty$ фуксову систему (3) с матрицей коэфбициентов вида

$$
B(z)=\frac{\left(\begin{array}{cccc}
* & 1 & 0 & 0 \\
* & * & \ddots & 0 \\
\vdots & \vdots & \ddots & 1 \\
* & * & \ldots & *
\end{array}\right)}{z}+\sum_{i=2}^{n-1} \frac{\left(\begin{array}{cccc}
* & 0 & 0 & 0 \\
* & * & \ddots & 0 \\
\vdots & \vdots & \ddots & 0 \\
* & * & \ldots & *
\end{array}\right)}{z-a_{i}}
$$

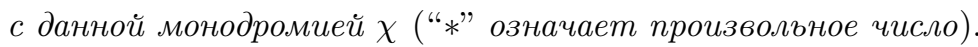

III. Построить голоморфное векторное расслоение $F$ с логарифмической

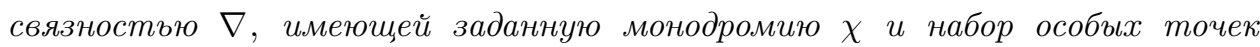
$a_{1}, \ldots, a_{n}$, образующее стабильную пару с типом расщепления $K=(0,(n-2)$, $\ldots,(n-2)(p-1))$.

Отметим, что фиксация особенностей $a_{1}$ и $a_{n}$ во второй задаче не ограничивает общности, так как этого всегда можно добиться дробно-линейной заменой переменной $z$, а такие замены сохраняют фуксовы особые точки.

2.3. Локальная структура фундаментальной системы решений. Мы рассмотрим в этом пункте фундаментальную систему решений системы (3) в окрестности регулярной особой точки $z=0$ (подробно об этом можно прочитать в [3]). В данном случае выбор точки не ограничит общности, так как с помощью подходящего дробно-линейного преобразования можно любую точку перевести в нуль.

Пусть фундаментальная матрица $Y(z)$ системы (3) имеет матрицу монодромии $G$ в точке $z=0$. Легко видеть, что матрица $z^{E}$, где $E$ - матрица, построенная по формуле

$$
E=\frac{1}{2 \pi i} \ln G, \quad \operatorname{spec}(E)=\left(\rho^{1}, \ldots, \rho^{p}\right) \quad(i=\sqrt{-1}),
$$

имеет в точке $z=0$ ту же матрицу монодромии $G$, что и $Y(z)$ :

$$
z^{E}=\widetilde{z^{E}} G, \quad Y(z)=\widetilde{Y}(z) G .
$$

Для определенности (однозначности матрицы $E$ ) фиксируем ветвь логарифма

$$
\rho^{k}=\frac{1}{2 \pi i} \ln \lambda^{k}, \quad 0 \leqslant \operatorname{Re} \rho^{k}<1 \quad(i=\sqrt{-1}),
$$

где $\lambda^{k}$ - собственные значения матрицы монодромии $G$. 
Нетрудно увидеть, что фундаментальная матрица $Y(z)$ может быть представлена в виде

$$
Y(z)=M(z) z^{E}
$$

с мероморфной в нуле матрицей $M(z)$.

На пространстве решений $X$ системы (3) можно ввести следующую функцию $\varphi(y)$, называемую нормированием. Каждому решению $y(z)$ функция $\varphi(y)$ ставит в соответствие целое число, равное минимуму целых частей показателей степеней в разложении (7) для данного решения. Так как решение $y(z)$ системы - это вектор-функция, то ее нормированием называется минимум нормирований ее компонент. Из разложения (7) видно, что решения раскладываются в ряды по степеням и логарифмам переменной $z$, поэтому определение корректно. Более того, нормирования решений - это целые части показателей асимптотик этих решений в точке $z=0$. Нормирование обладает следующими свойствами.

ПредЛОжениЕ 1. Нормирование $\varphi$ задает отображение $\varphi: X \rightarrow \mathbb{Z} \cup\{\infty\}$, обладающее следующими свойствами:

а) $\varphi\left(y_{1}+y_{2}\right) \geqslant \min \left(\varphi\left(y_{1}\right), \varphi\left(y_{2}\right)\right)$, причем если $\varphi\left(y_{1}\right) \neq \varphi\left(y_{2}\right)$, то имеет место равенство;

б) $\varphi($ су $)=\varphi(y)$ для любого $c \in \mathbb{C} \backslash\{0\}$;

в) $\varphi\left(\sigma^{*} y\right)=\varphi(y)$, где $\sigma^{*}$ обозначает действие оператора монодромии в точке нуль.

Доказательство этого предложения нетрудно вывести из определения нормирования и вида (7) решений в окрестности точки нуль.

Из свойств а) и б) следует, что нормирование $\varphi$ принимает на $X$ конечное число значений $\infty>\psi_{1}>\cdots>\psi_{m}$ и задает фильтрацию

$$
0 \subset X_{1} \subset \cdots \subset X_{m}=X
$$

пространства $X$ линейными подпространствами

$$
X_{k}=\left\{y \in X \mid \varphi(y) \geqslant \psi_{k}\right\}, \quad k=1, \ldots, m .
$$

По свойству в) оператор монодромии $\sigma^{*}$ сохраняет эту фильтрацию. Обозначим через $k_{l}$ размерность факторпространства $X_{l} / X_{l-1}$, а через $\sigma_{l}^{*}$ - ограничение $\sigma^{*}$ на $X_{l}$.

Рассмотрим базис $e_{1}^{1}, \ldots, e_{k_{1}}^{1}$ пространства $X_{1}$, в котором матрица оператора $\sigma_{1}^{*}$ имеет верхнетреугольный вид, дополним его до базиса $e_{1}^{1}, \ldots, e_{k_{1}}^{1}, e_{1}^{2}, \ldots, e_{k_{2}}^{2}$ пространства $X_{2}$, в котором верхнетреугольный вид имеет матрица оператоpa $\sigma_{2}^{*}$, и т. д. Построенный базис $(e)=\left(e_{1}, \ldots, e_{p}\right)$ пространства $X$ обладает следующими свойствами:

1) нормирование $\varphi$ принимает на элементах базиса $(e)$ все свои значения $\psi_{1}, \ldots, \psi_{m}$ (с учетом кратностей $\left.k_{1}, \ldots, k_{m}\right)$;

2) $\varphi\left(e_{l+1}\right) \leqslant \varphi\left(e_{l}\right), l=1, \ldots, p-1$;

$3)$ матрица $G$ оператора $\sigma^{*}$ имеет в этом базисе верхнетреугольный вид.

Базис $(e)$ пространства $X$ решений системы (3) в окрестности регулярной особой точки $z=0$, удовлетворяющий свойствам 1)-3), называют левелевским. 
Для фундаментальной матрицы $Y(z)$ системы (3), построенной по левелевскому базису, имеет место локальное представление в виде произведения трех матриц определенного вида:

$$
Y(z)=U(z) z^{A} z^{E}, \quad A=\left(\begin{array}{ccc}
\varphi^{1} & & 0 \\
& \ddots & \\
0 & & \varphi^{p}
\end{array}\right), \quad E=\left(\begin{array}{ccc}
\rho^{1} & * & * \\
& \ddots & * \\
0 & & \rho^{p}
\end{array}\right),
$$

где $U(z)$ - голоморфная в нуле матрица, каждый столбец которой имеет хотя бы один элемент, не обращающийся в нуль при $z=0$. Диагональные элементы $\varphi^{k}$ - целые числа (целые части асимптотик решений), для которых выполнено условие

$$
\varphi^{1} \geqslant \cdots \geqslant \varphi^{p}
$$

а $\rho^{k}$ - нормализованные логарифмы собственных значений матрицы $G$ монодромии в нуле, удовлетворяющие условиям (6).

Отметим, что для произвольной фундаментальной матрицы $Y(z)$, не обязательно левелевской, имеется следующее представление:

$$
Y(z)=U(z) z^{A} z^{E} S
$$

с некоторой постоянной невырожденной матрицей $S$.

Такое же разложение верно и в случае скалярных фуксовых уравнений (1), с тем изменением, что фундаментальная система решений $Y(z)$ - вектор-строка и $U(z)$ - тоже голоморфная вектор-строка, элементы которой не обращаются в нуль в нуле.

Описанный выше базис пространства решений, называемый левелевским, всегда существует. Наряду с левелевским базисом также можно рассмотреть базис, который получается как объединение левелевских базисов корневых (для оператора монодромии) подпространств - он называется слабо левелевским. Если снять условие (9) и условие верхнетреугольности матрицы $E$, но наложить условие, что нормирование на данном базисе принимает все свои значения с учетом кратности или что матрица $z^{A} E z^{-A}$ голоморфна в нуле, то базис называют ассоциированным. Можно показать, что левелевский и слабо левелевский базисы всегда существуют и являются ассоциированными (см. [3]).

Матрицу коэффициентов системы (3) в окрестности регулярной особой точки $z=0$ можно записать в виде

$$
B(z)=\frac{d U}{d z} U^{-1}+U(z) \frac{A+z^{A} E z^{-A}}{z} U^{-1}(z) .
$$

Величину $\beta^{j}=\varphi^{j}+\rho^{j}$ называют показателем. Из (11) видно, что показатели суть собственные числа вычетов матрицы коэффициентов системы.

Теорема 5 (А. Левель, см. [3]). Система (3) фуксова в точке $z=0$ тогда и только тогда, когда матрица $U(z)$ из разложения (8) голоморфно обратима в $z=0, m$.e. $\operatorname{det} U(0) \neq 0$. 
Достаточность будет следовать из вида (11) матрицы коэффициентов системы (3) при учете вида разложения (8). Полное доказательство см. в [3; теорема 5.2].

Легко видеть, что разложение (8) и теорема Левеля дают вид локальных нормальных форм системы (3) в окрестности регулярных и фуксовых особенностей относительно голоморфных и мероморфных калибровочных преобразований. Опишем их в виде следующей теоремы.

ТЕОРема 6. (I) Систему (3) с помощью голоморфного в окрестности фуксовой особой точки $z=0$ калибровочного преобразования можно привести к системе вида

$$
y^{\prime}=\frac{A+z^{A} E z^{-A}}{z} y,
$$

причем в нерезонансном случае она имеет вид $y^{\prime}=\frac{A+E}{z} y$ (резонансной называется точка, где какая-нибудь пара показателей асимптотик отличается на целое положительное число).

(II) В окрестности фуксовой или регулярной особой точки $z=0$ система (3) мероморфным преобразованием приводится к виду

$$
y^{\prime}=\frac{E}{z} y .
$$

Далее, когда речь пойдет о точке $z=a_{i}$, у всех матриц появится нижний индекс " $i$ ", указывающий на рассмотрение в окрестности точки $a_{i}$.

\section{4. Векторные расслоения с логарифмическими связностями над} сферой. В этом пункте мы рассмотрим голоморфные векторные расслоения с логарифмическими связностями над сферой Римана. Задавать расслоение со связностью будем с помощью координатного описания.

Говорят, что заданы локальные тривиализации расслоения, если заданы следующие три составляющие:

a) покрытие $\left\{U_{i}\right\}$ базы $\mathbf{B}(\mathbf{B}=\overline{\mathbb{C}})$ открытыми окрестностями такое, что над каждой $U_{i}$ расслоение $F$ тривиально;

б) набор голоморфных невырожденных матричных функций

$$
g_{i j}: U_{i} \cap U_{j} \rightarrow \mathrm{GL}(p, \mathbb{C}),
$$

определенных в пересечениях окрестностей, удовлетворяющих условиям леммы 1 (см. ниже); набор таких функций называют коииклом;

в) фиксированы локальные тривиализации $s_{i}$ расслоения $F$ над окрестностями $U_{i}$, т. е. над каждой окрестностью $U_{i}$ фиксирован набор

$$
\left(s_{i}\right)=\left(s_{i}^{1}(z), \ldots, s_{i}^{p}(z)\right)
$$

из $p$ локальных голоморфных сечений (вектор-функций $s_{i}^{j}(z): U_{i} \rightarrow \mathbb{C}^{p}$ ), образующих в каждой точке $z$ окрестности $U_{i}$ базис $\left(s_{i}^{1}(z), \ldots, s_{i}^{p}(z)\right)$ в слое $\mathbf{L}=\mathbb{C}^{p}$.

Пусть окрестности $U_{i}, U_{j}$ имеют непустое пересечение. Тогда определим функцию $g_{i j}(z)$ как функцию перехода между тривиализациями $\left(s_{i}\right)=g_{i j}\left(s_{j}\right)$. 
ЛЕмма 1. По векторному расслоению $F$, покрытию $\left\{U_{i}\right\}$ и фиксированным тривиализачиям $\left\{\left(s_{i}\right)\right\}$ расслоения $F$ над $\left\{U_{i}\right\}$ можно построить набор отображений (12), обладающих следующими свойствами:

$$
\begin{array}{ll}
g_{i j}(z) \equiv\left(g_{j i}(z)\right)^{-1}, & z \in U_{i} \cap U_{j} ; \\
g_{j i}(z) g_{i k}(z) g_{k j}(z) \equiv I, & z \in U_{i} \cap U_{j} \cap U_{k} .
\end{array}
$$

И обратно, по любому покрытию $\left\{U_{i}\right\}$ многообразия $\mathbf{B}$ и по любому набору отображений (12), обладающих свойствами (13), можно построить векторное расслоение $F^{\prime}=\left(\mathbf{L}, \mathbf{B}, \widetilde{\pi}^{\prime}\right)$. Если набор отображений $\left\{g_{i j}\right\}$ был построен по векторному расслоению $F$, то расслоение $F^{\prime}$ будет эквивалентно расслоению $F$.

При таком описании тотальное пространство $\mathbf{F}$ не фигурирует явно. Чтобы определить пространство $\mathbf{F}$, рассмотрим несвязное объединение

$$
\widetilde{F}=\bigsqcup U_{i} \times \mathbb{C}^{p}
$$

и введем на множестве $\widetilde{F}$ следующее отношение эквивалентности $\sim$ : если $x \in$ $U_{i} \cap U_{j}$, то

$$
\begin{aligned}
(x, v) & \sim\left(x, g_{j i}(x) v\right), & & (x, v) \in U_{i} \times \mathbb{C}^{p}, \\
(x, v) & \sim(x, v), & & x \in U_{i}, \quad v \in \mathbb{C}^{p} .
\end{aligned}
$$

Из свойств (13) следует, что отношение эквивалентности определено корректно и $\mathbf{F}=\widetilde{F} / \sim$. Подробнее об этом можно прочитать в [3; лекция 2].

Говорят, что в векторном расслоении $F$ задана связность $\nabla$, если для любой тривиализации расслоения (т. е. покрытия $\left\{U_{i}\right\}$, набора коциклов $\left\{g_{i j}(z)\right\}$ и $\left.\left\{\left(s_{i}\right)\right\}\right)$ задан набор матричных дифференциальных 1-форм $\left\{\omega_{i}\right\}$, удовлетворяющих соотношениям

$$
\omega_{i}=\left(d g_{i j}\right) g_{i j}^{-1}+g_{i j} \omega_{j} g_{i j}^{-1}
$$

Все пары $(F, \nabla)$ (расслоение со связностью) допускают координатное описание, задается покрытие сферы Римана окрестностями $\left\{U_{i}\right\}$, над каждой из которых расслоение тривиально. Связность в этой окрестности определяется как система линейных дифференциальных уравнений

$$
d y=\omega_{i} y, \quad z \in U_{i}
$$

Базис горизонтальных сечений $\left(s_{i}\right)$ связности - это фундаментальная система решений этой системы, которая может быть выбрана в виде (10). Связность называют логарифмической или фуксовой, если определяющие ее системы (15) фуксовы в своих окрестностях. Базисы пространств решений систем, заданных в соседних окрестностях, отождествляются по формуле $\left(s_{i}\right)=g_{i j}(z)\left(s_{j}\right)$ с помощью склеивающих функций $g_{i j}(z)$, определенных в пересечениях $U_{i} \cap U_{j}$ и образующих голоморфный коцикл. Благодаря теореме 6 мы имеем локальную аналитическую классификацию линейных систем в окрестности фуксовой 
особой точки. Благодаря этому можно дать классификацию всех голоморфных расслоений с логарифмическими связностями на сфере Римана, с точностью до голоморфной эквивалентности расслоений и связностей. Получается, что пара $(F, \nabla)$ - расслоение с логарифмической связностью - однозначно определяется представлением монодромии (2) и локальными базисами горизонтальных сечений, которые определяются наборами матриц

$$
A=\left\{A_{1}, \ldots, A_{n}\right\} \quad \text { и } \quad S=\left\{S_{1}, \ldots, S_{n}\right\} .
$$

Будем обозначать такие пары через $\left(F^{A, S}, \nabla^{A, S}\right)$. Подробно об этом см. в [3].

2.5. Глобальная классификация голоморфных расслоений над $\overline{\mathbb{C}}$. Каждое голоморфное расслоение на сфере Римана, согласно теореме Биркгофа-Гротендика, эквивалентно некоторому расслоению вида

$$
\left(U_{0}=\mathbb{C}, U_{\infty}=\overline{\mathbb{C}} \backslash\{0\}, g_{0 \infty}=z^{K}\right), \quad K=\operatorname{diag}\left(k_{1}, \ldots, k_{p}\right),
$$

где $k_{1} \leqslant \cdots \leqslant k_{p}$ (заметим, что в цитированной литературе обычно рассматривается обратный порядок следования элементов). Другими словами, любое голоморфное расслоение над сферой Римана эквивалентно прямой сумме одномерных расслоений

$$
\widetilde{F} \cong \mathscr{O}\left(k_{1}\right) \oplus \cdots \oplus \mathscr{O}\left(k_{p}\right)
$$

вида

$$
\mathscr{O}\left(k_{i}\right)=\left(U_{0}=\mathbb{C}, U_{\infty}=\overline{\mathbb{C}} \backslash\{0\}, g_{0 \infty}=z^{k_{i}}\right) .
$$

Набор целых чисел $\left(k_{1}, \ldots, k_{p}\right)$ называют типом расщепления. Степенъю расслоения называют число

$$
\operatorname{deg} F=\sum_{i=1}^{p} k_{i}
$$

а наклоном - величину

$$
\mu(F)=\frac{\operatorname{deg} F}{\operatorname{rk} F} .
$$

Кроме того, известно, что степень расслоения, снабженного логарифмической связностью, равна сумме показателей: $\operatorname{deg} F=\sum_{i=1}^{n} \sum_{j=1}^{p} \beta_{i}^{j}$, где $\beta_{i}^{j}-$ собственные значения вычетов связности в особой точке $a_{i}$, они же асимптотики решений ассоциированного базиса.

Расслоение $F$ называется стабилъным (полустабильным), если для любого его подрасслоения $F_{\text {sub }}$ выполнено неравенство $\mu(F)>\mu\left(F_{\text {sub }}\right)$ (неравенство $\left.\mu(F) \geqslant \mu\left(F_{\mathrm{sub}}\right)\right)$. Стабильных расслоений над сферой не бывает, но для нас будет важно понятие стабильного расслоения со связностью.

Подрасслоение называют стабилизирующимся связностъю, если подпространство его горизонтальных сечений является инвариантным относительно действия монодромии связности. Расслоение $F$ со связностью $\nabla$ называют стабильной парой, если для любого его подрасслоения $F_{\mathrm{sub}}$, которое стабилизируется связностью, выполнено соотношение $\mu(F)>\mu\left(F_{\mathrm{sub}}\right)$. 
Рассмотрим расслоение $F$ с типом расщепления $\left(k_{1}, \ldots, k_{p}\right)$, тогда фуксовым весом расслоения $F$ назовем величину

$$
\gamma(F)=\sum_{i=1}^{p}\left(k_{p}-k_{i}\right) .
$$

Теперь рассмотрим множество всех расслоений с логарифмическими связностями $\left(F^{A, S}, \nabla^{A, S}\right)$, имеющих одно и то же представление монодромии (2). Тогда максимальным фуксовым весом представления (2) называют величину

$$
\gamma_{\max }(\chi)=\max _{A, S} \gamma\left(F^{A, S}\right)
$$

В [5] можно найти следующую теорему.

Теорема 7. Максималъный фуксов вес представления (2) существует (не равен бесконечности) тогда и только тогда, когда оно неприводимо. Для неприводимого представления существует следующая оченка для фуксова веса:

$$
\gamma_{\max }(\chi) \leqslant \frac{(n-2) p(p-1)}{2},
$$

где $n$ - число образующих представления, а $p$ - его размерность.

Далее мы, как правило, будем считать, что $a_{1}=0, a_{n}=\infty$. Это предположение не ограничивает общности, так как этого всегда можно добиться дробно-линейной заменой независимой переменной. Как известно, такие замены переводят фуксовы точки в фуксовы. Везде предполагается, что число особых точек $n$ не меньше 3 . Случай $n=2$ разбирается тривиально, но формально не все формулировки будут верны, так как в этом случае не бывает стабильных пар. Введем полезное обозначение:

$$
D=\operatorname{diag}(0,1, \ldots, p-1) .
$$

Сразу оговоримся, что в разных разделах и доказательствах теорем одни и те же обозначения могут обозначать разные объекты.

\section{3. Доказательство теоремы 4}

В этом разделе содержатся три теоремы, доказывающие в совокупности теорему 4, которая заключается в утверждении об эквивалентности трех формулировок 21-й проблемы Гильберта для скалярных фуксовых уравнений:

$$
\begin{aligned}
& \text { T8: III } \Longrightarrow \text { II, } \\
& \text { T9: } \mathrm{II} \Longrightarrow \mathrm{I} \text {, } \\
& \text { T10 : } \mathrm{I} \Longrightarrow \text { III }
\end{aligned}
$$

ТЕорема 8. По представлению монодромии (2) и набору особых точек

$$
a_{1}=0, a_{2}, \ldots, a_{n-1}, a_{n}=\infty, \quad n \geqslant 3,
$$


стабильной парь $(F, \nabla)$, имеющей тип расщепления

$$
K=\operatorname{diag}(0,(n-2), \ldots,(n-2)(p-1))=(n-2) D,
$$

можно построить фуксову систему (3) с матрицей коэфбициентов вида (5).

ДокАзАтельство. Рассмотрим пару $(F, \nabla)$ (расслоение со связностью), заданную по условию теоремы. Представим ее в координатной форме. Рассмотрим две координатные окрестности: $U_{0}$ - малая окрестность нуля, не содержащая других особых точек, и $U_{\infty}=\overline{\mathbb{C}} \backslash\{0\}$, а также коцикл, задаваемый склеивающей функцией

$$
g_{0 \infty}(z)=z^{K}=z^{(n-2) D}
$$

Тройка $\left(U_{0}, U_{\infty}, g_{0 \infty}\right)$ определяет расслоение $F$. Связность задают две формы $\omega_{0}$ и $\omega_{\infty}$, которые фуксовы в $U_{0}$ и $U_{\infty}$ соответственно. Эти формы связаны соотношением

$$
\omega_{0}=g_{0 \infty} \omega_{\infty} g_{0 \infty}^{-1}+d g_{0 \infty} \cdot g_{0 \infty}^{-1} .
$$

Из (16) следует, что для элементов $\omega_{k l}^{0}(z)$ и $\omega_{k l}^{\infty}(z)$ матричных форм $\omega_{0}$ и $\omega_{\infty}$ выполнены следующие соотношения:

$$
\omega_{k l}^{\infty}(z)=z^{(n-2)(l-k)} \omega_{k l}^{0}(z), \quad k \neq l .
$$

Отсюда, а также из того, что формы $\omega_{0}$ и $\omega_{\infty}$ имеют лишь простые полюсы в точках $a_{1} \in U_{0}$ и $a_{2}, \ldots, a_{n} \in U_{\infty}$ соответственно, следует, что элементы форм $\omega_{k l}^{0}(z)$ и $\omega_{k l}^{\infty}(z)$ с номерами $(k, l)$ для $l-k>1$ тождественно равны нулю. Действительно, соотношение (17) и условие $l-k>1$ дают то, что элемент $\omega_{k l}^{\infty}(z)$ имеет нуль порядка

$$
\operatorname{ord}_{0} \omega_{k l}^{\infty}(z)=(n-2)(l-k)-1 \geqslant n-2
$$

в нуле, а полюсы у $\omega_{k l}^{\infty}(z)$ есть лишь в точках $a_{2}, \ldots, a_{n}$ и имеют первый порядок, т. е.

$$
\operatorname{ord}_{a_{i}} \omega_{k l}^{\infty}(z) \geqslant-1, \quad i=2, \ldots, n .
$$

При этом разность суммы порядков нулей и суммы порядков полюсов дифференциальной формы на сфере Римана равна -2 (если она не является тождественно нулевой), а у элемента $\omega_{k l}^{\infty}(z)$ эта разность должна быть не меньше, чем

$$
\operatorname{ord}_{0} \omega_{k l}^{\infty}(z)+\sum_{i=2}^{n} \operatorname{ord}_{a_{i}} \omega_{k l}^{\infty}(z) \geqslant-1>-2 .
$$

Следовательно,

$$
\omega_{k l}^{\infty}(z) \equiv 0 \quad \text { при } l-k>1 .
$$

Таким образом, наше расслоение со связностью заданы двумя почти треугольными формами $\omega_{0}$ и $\omega_{\infty}$ и коциклом $g_{0 \infty}(z)$. Вместо форм $\omega_{0}$ и $\omega_{\infty}$ нам удобно будет рассмотреть форму

$$
\omega=\Gamma(z) \omega_{\infty} \Gamma^{-1}(z)+\frac{d \Gamma}{d z} \Gamma^{-1}, \quad \Gamma(z)=\prod_{i=2}^{n-1}\left(\frac{z}{z-a_{i}}\right)^{D} .
$$


Далее будем рассматривать регулярную систему

$$
d y=\omega y
$$

Эта система имеет фуксовы особенности в нуле и бесконечности и регулярные в точках $a_{2}, \ldots, a_{n-1}$. Покажем, что

$$
\omega=\sum_{i=2}^{n-1} \frac{\left(\begin{array}{cccc}
* & 0 & 0 & 0 \\
* & * & 0 & 0 \\
* & \alpha_{k l}^{i} & * & 0 \\
* & * & * & *
\end{array}\right)}{z-a_{i}} d z+\frac{\left(\begin{array}{cccc}
* & 1 & 0 & 0 \\
* & * & 1 & 0 \\
* & * & * & 1 \\
* & * & * & *
\end{array}\right)}{z} d z
$$

Из вида преобразования (18) следует, что функции $\alpha_{k l}^{i}(z)$ имеют полюсы порядков не выше $k-l$ в точке $a_{i}$ :

$$
\operatorname{ord}_{a_{i}} \alpha_{k l}^{i}(z) \geqslant l-k,
$$

и голоморфны везде вне $z=a_{i}, i=2, \ldots, n-1$, в частности, наддиагональные элементы получаются нулевыми везде, кроме последнего члена. То, что наддиагональ последнего коэффициента единичная, не следует из сказанного выше, но в случае, когда ни один ее элемент не равен нулю, этого можно достичь подходящим постоянным диагональным калибровочным преобразованием. Случай, когда какой-то из наддиагональных элементов нулевой, $\omega_{l, l+1}(z) \equiv 0$, противоречит условию стабильности исходной пары $(F, \nabla)$. Это будет сейчас показано.

Действительно, в этом случае расслоение и связность приводятся. Подрасслоение, которое стабилизируется связностью, соответствует нижнему правому блоку форм связности и коцикла, но легко видеть, что тип расщепления $K_{1}$ этого подрасслоения $F_{1}$ совпадает с концом типа расщепления расслоения $F$, т. е. состоит из наибольших его элементов: $K_{1}=\left(k_{l+1}, \ldots, k_{p}\right)$. Тогда его наклон больше наклона $F$ :

$$
\mu\left(F_{1}\right)=\frac{k_{l+1}+\cdots+k_{n}}{n-l}=\frac{(n-2)(p+l-1)}{2}=\frac{(n-2)(p-1)}{2}>\mu(F)
$$

- мы получили противоречие с условием стабильности пары $(F, \nabla)$. Таким образом, доказано, что форма $\omega$ имеет вид (19).

Калибровочное преобразование $\tilde{y}=\left(z-a_{i}\right)^{D} y$ переводит систему (19) в фуксову в точке $a_{i}\left(d \tilde{y}=\omega_{i} \tilde{y}\right)$, причем вычет матрицы коэффициентов преобразованной системы в точке $z=a_{i}$ имеет вид

$$
\left(\begin{array}{cccc}
* & a_{i}^{-1} & 0 & 0 \\
* & * & a_{i}^{-1} & 0 \\
* & * & * & a_{i}^{-1} \\
* & * & * & *
\end{array}\right)
$$

где “*” обозначают некоторые числа. Следовательно, фундаментальная матрица системы (19) в точке $a_{i}$ имеет вид

$$
Y(z)=\left(z-a_{i}\right)^{-D} Y_{i}^{0}(z)
$$


где матрица $Y_{i}^{0}(z)$ - это фундаментальная матрица фуксовой в $z=a_{i}$ системы. Продолжая, получим представление

$$
Y(z)=T_{i}(z)\left(z-a_{i}\right)^{-D} Y^{0 \prime}(z), \quad T_{i}(z)=\left(\prod_{j=2}^{n-1}\left(z-a_{j}\right)^{-D}\right)\left(z-a_{i}\right)^{D}
$$

где $Y^{0}(z)$ - фундаментальная матрица фуксовой в точках $a_{2}, \ldots, a_{n-1}$ системы.

Далее нам потребуется лемма 2, которая приведена ниже. Согласно этой лемме, применив калибровочное преобразование

$$
\tilde{y}=\Gamma_{i}(z) y
$$

к фундаментальной матрице $\left(z-a_{i}\right)^{-D} Y^{0 \prime}(z)$, мы получим фундаментальную матрицу фуксовой в точке $z=a_{i}$ системы. Но, поскольку матрица (22) имеет еще один множитель, нужно видоизменить преобразование из леммы 2 и рассмотреть сопряженное к нему:

$$
\tilde{y}=T_{i} \Gamma_{i} T_{i}^{-1} y
$$

Сопряженное преобразование $\widetilde{\Gamma}_{i}=T_{i} \Gamma_{i} T_{i}^{-1}$, так же как исходное, голоморфно обратимо вне точки $z=a_{i}$, это видно из нижнетреугольности матрицы $\Gamma_{i}(z)$ и вида матрицы $T_{i}(z)$.

Тогда мы можем применять преобразования $y^{\prime}=\widetilde{\Gamma}_{i} y$ последовательно для всех точек $a_{2}, \ldots, a_{n-1}$ и получим фундаментальную матрицу системы

$$
Y^{\prime}(z)=\widetilde{\Gamma}_{2}(z) \cdots \widetilde{\Gamma}_{n-1}(z) Y(z) .
$$

Полученная система и будет искомой системой вида (3), (5).

Лемма 2. Существует нижнетреугольная матрица $\Gamma_{i}(z)$, голоморфно обратимая в $\overline{\mathbb{C}} \backslash\left\{a_{i}\right\}$ и мероморфная в $a_{i}\left(\Gamma_{i}(z)\right.$ - матричный многочлен от $1 / z$ с постоянными ненулевыми диагональными элементами), при умножении на которую фундаментальная матрища (21) преобразуется в матрицу

$$
Y_{i}^{\prime}(z)=\Gamma_{i}(z) Y_{i}(z)=\Gamma_{i}(z)\left(z-a_{i}\right)^{-D} Y_{i}^{0}(z)=U_{i}^{\prime}(z)\left(z-a_{i}\right)^{\Lambda_{i}-D}\left(z-a_{i}\right)^{E_{i}},
$$

где $Y_{i}^{\prime}(z)$ - фундаментальная матрица фуксовой в $z=a_{i}$ системъ, т.е. $\operatorname{det} U_{i}^{\prime}(0) \neq 0$.

ДокАЗАТЕЛЬСтво. Будем строить преобразование $\Gamma_{i}(z)$ в виде цепочки элементарных преобразований. Рассмотрим преобразования вида

$$
\Gamma_{i}^{\alpha \beta}(z)=I+E_{\alpha \beta}, \quad \alpha>\beta,
$$

где $E_{\alpha \beta}$ содержит единственный ненулевой элемент $e_{\alpha \beta}(z)$, стоящий на месте $(\alpha, \beta)$, который является многочленом степени $\alpha-\beta$ от $1 / z$, причем для элементов матрицы $U_{i}(z)$ выполнено

$$
u_{\alpha \beta}(z)-e_{\alpha \beta}(z) z^{\alpha-\beta} u_{\beta \beta}(z)=z^{\alpha-\beta} h_{\alpha \beta}(z)
$$


с некоторой голоморфной функцией $h_{\alpha \beta}(z)$. Заметим, что это возможно всегда, когда $u_{\beta \beta}\left(a_{i}\right) \neq 0$. Для того чтобы показать это, нам понадобится лемма 3. Действительно, из леммы 3 следует, что $u_{11}\left(a_{i}\right) \neq 0$, так как этот элемент совпадает с первым главным минором. Это позволяет применить преобразования $\Gamma_{i}^{\alpha 1}(z), \alpha=2, \ldots, p$, причем эти преобразования, как нижнетреугольные с единичной диагональю, не изменяют главных угловых миноров. Отсюда следует, что элемент $u_{22}^{\prime}\left(a_{i}\right)$ преобразованной матрицы отличен от нуля, и мы можем продолжить применять преобразования. Таким образом, мы получим искомое преобразование $\Gamma_{i}(z)$ в виде произведения элементарных преобразований. Лемма 2 доказана.

Лемма 3. Все главные угловые миноры матрии $U_{i}\left(a_{i}\right)$ системы $(3),(5)$ при $i=2, \ldots, n-1$ отличны от нуля.

Для доказательства этой леммы нам понадобится конструкция мероморфного калибровочного преобразования, преобразующего регулярную систему с рациональными коэффициентами к системе, эквивалентной фуксову уравнению.

(А) Преобразование системы к уравнению. Воспользуемся рассуждением, аналогичным рассуждению из [11], переведенному на язык аналитической теории дифференциальных уравнений.

Рассмотрим регулярную систему вида (3) с рациональной матрицей коэффициентов $B(z)$. Возьмем $p$-мерную вектор-строку $t_{0}=(1,0, \ldots, 0)$. Далее определим мероморфные на сфере Римана вектор-функции $t_{1}(z), \ldots, t_{p}(z)$ формулами

$$
t_{j+1}=\frac{d t_{j}}{d z}+t_{j} B(z), \quad j=0, \ldots, p-1 .
$$

Рассмотрим матрицу $\Gamma(z)$, строками которой являются $t_{0}, t_{1}(z), \ldots, t_{p-1}(z)$. Очевидно, что если у строки $t_{0}$ отличен от нуля только первый элемент, то, поскольку матрица $B(z)$ коэффициентов системы имеет почти треугольный вид (5), матрица $\Gamma(z)$ оказывается нижнетреугольной. Из не равенства нулю наддиагонали вычета (20) и формулы (23) получим, что на диагонали у $\Gamma(z)$ стоят элементы $\gamma_{i i}(z)$, имеющие полюсы порядка $i-1$ в точках $a_{2}, \ldots, a_{n-1}$.

Теперь покажем, что преобразование $\tilde{y}=\Gamma(z) y$ переводит систему в систему, эквивалентную скалярному уравнению. Рассмотрим вектор-функцию $b(z)=$ $\left(b_{p}(z), \ldots, b_{1}(z)\right)$, удовлетворяющую соотношению

$$
t_{p}(z)=-b(z) \Gamma(z)
$$

и соответствующую ей матрицу

$$
\widetilde{B}(z)=\left(\begin{array}{cccc}
0 & 1 & & 0 \\
\vdots & \ddots & \ddots & \\
0 & \ldots & 0 & 1 \\
-b_{p} & \cdots & \ldots & -b_{1}
\end{array}\right)
$$

Из формул (23) следует, что

$$
\frac{d \Gamma}{d z}=\widetilde{B} \Gamma-\Gamma B
$$


поэтому преобразование $\tilde{y}=\Gamma(z) y$ переводит систему (3) в систему с матрицей коэффициентов

$$
\Gamma B \Gamma^{-1}+\frac{d \Gamma}{d z} \Gamma^{-1}=\Gamma B \Gamma^{-1}+\widetilde{B}-\Gamma B \Gamma^{-1}=\widetilde{B}
$$

искомого вида.

ДокАЗАТЕЛЬСтво лЕммы 3. Рассмотрим построенное выше калибровочное преобразование $\tilde{y}=\Gamma(z) y$, которое приводит систему к виду, эквивалентному уравнению.

Теперь рассмотрим преобразование $\widetilde{\Gamma}_{i}(z)=\left(z-a_{i}\right)^{D} \Gamma$, которое приводит систему к системе с матрицей коэффициентов вида

$$
\widetilde{B}(z)=\left(\begin{array}{cccc}
0 & 1 & 0 & 0 \\
\vdots & 1 & \ddots & 0 \\
0 & \ldots & \ddots & 1 \\
0 & \ldots & 0 & p-1
\end{array}\right) \frac{1}{z-a_{i}}+\left(\begin{array}{cccc}
0 & 0 & \ldots & 0 \\
\vdots & \ddots & \ddots & \vdots \\
0 & \ldots & 0 & 0 \\
-\tilde{b}_{p} & \ldots & \ldots & -\tilde{b}_{1}
\end{array}\right)
$$

где $\tilde{b}_{k}=\left(z-a_{i}\right)^{k-1} b_{k}, k=2, \ldots, n-1$, которая сводится к уравнению (1) с помощью замены

$$
y^{1}=y, \quad y^{2}=\left(z-a_{i}\right) y^{\prime}, \quad \ldots, \quad y^{p}=\left(z-a_{i}\right)^{p-1} y^{(p-1)} .
$$

Заметим, что из вида формы (19) коэффициентов исходной системы и способа построения матриц $\Gamma(z)$ и $\Gamma_{i}(z)$ (из п. (А) выше) калибровочных преобразований следует, что преобразование $\widetilde{\Gamma}_{i}(z)$ нижнетреугольное, с единичной диагональю и голоморфно обратимое в $z=a_{i}$. Такое преобразование не может изменить главных угловых миноров матрицы $U_{i}\left(a_{i}\right)$, т. е. они равны главным угловым минорам матрицы $\widetilde{\Gamma}_{i}\left(a_{i}\right) U_{i}\left(a_{i}\right)$.

Для системы, полученной из фуксова уравнения (1) с помощью замены вида (26), известно (см., например, [5]), что ее главные угловые миноры отличны от нуля.

В самом деле, рассмотрим ассоциированный базис решений фуксова в $z=a_{i}$ уравнения, состоящий из решений $u_{1}(z), \ldots, u_{p}(z)$ и такой, что матрица монодромии $G_{i}$ в точке $z=a_{i}$ имеет верхнетреугольный вид, например левелевский. Тогда определитель матрицы $U_{i}\left(a_{i}\right)$, полученной из уравнения $(1)$ с помощью замены (26), отличен от нуля по теореме Левеля, так как эта система (3), (25) фуксова в точке $z=a_{i}$. Теперь рассмотрим набор функций $u_{1}(z), \ldots, u_{k}(z)$, $k<p$. Этот набор тоже является ассоциированным базисом решений уравнения

$$
\frac{1}{W\left(u_{1}, \ldots, u_{k}\right)} \operatorname{det}\left(\begin{array}{cccc}
u_{1} & \ldots & u_{k} & y \\
\frac{d u_{1}}{d z} & \ldots & \frac{d u_{k}}{d z} & \frac{d y}{d z} \\
\vdots & \ddots & \vdots & \vdots \\
\frac{d^{k} u_{1}}{d z^{k}} & \ldots & \frac{d^{k} u_{k}}{d z^{k}} & \frac{d^{k} y}{d z^{k}}
\end{array}\right)=0
$$


Это уравнение фуксово (по теореме Фукса), так как оно регулярно. Это означает, что с помощью аналогичной (26) замены из него можно получить фуксову в $z=a_{i}$ систему. У этой системы будет соответствующая матрица $U_{i}^{k}\left(a_{i}\right)$, определитель которой отличен от нуля по теореме Левеля. Заметим, что из вида замены $(26)$ и из левелевского разложения (8) видно, что эта матрица $U_{i}^{k}\left(a_{i}\right)$ подматрица матрицы $U_{i}\left(a_{i}\right)$, стоящая на пересечении первых $k$ строк и первых $k$ столбцов. Таким образом, главные угловые миноры $U_{i}\left(a_{i}\right)$ - это определители соответствующих матриц систем меньших размерностей, и, соответственно, все они отличны от нуля. Лемма доказана.

ТеОрема 9. Если по монодромии $\chi$ и набору особых точек $a_{1}, \ldots, a_{n}$ можно построить систему вида (3), (5), то можно построить и фуксово уравнение (4).

ДокАЗАтЕльство. Доказательство состоит в последовательном применении мероморфных калибровочных преобразований, приводящих в итоге систему вида (3), (5) к системе с матрицей коэффициентов вида (24), эквивалентной скалярному уравнению. Легко убедиться, что эти калибровочные преобразования не добавят новых особых точек, а то, что полученное в итоге уравнение будет фуксовым, следует из его регулярности, которая очевидным образом вытекает из регулярности исходной фуксовой системы.

Рассмотрим преобразование вида

$$
\tilde{y}=\Gamma_{\alpha \beta}^{i} y, \quad \Gamma_{\alpha \beta}^{i}(z)=I+E_{\alpha \beta}^{i}(z), \quad \alpha>\beta,
$$

с матрицами $E_{\alpha \beta}^{i}(z)$, содержащими только один ненулевой элемент

$$
\begin{aligned}
e_{\alpha \beta}^{i}(z) & =\sum_{k=1}^{\alpha-\beta} h_{\alpha \beta}^{i k} \frac{z}{\left(z-a_{i}\right)^{k}}, \quad i=2, \ldots, n-1, \\
e_{\alpha \beta}^{1}(z) & =\text { const }
\end{aligned}
$$

на месте $(\alpha, \beta)$ с подходящими постоянными $h_{\alpha \beta}^{i k}$. Матрицы преобразований $\Gamma_{\alpha \beta}^{i}(z)$ голоморфно обратимы вне $a_{i}$ при $i=2, \ldots, n-1$ и постоянны при $i=1$. Система под действием этих преобразований перестанет быть фуксовой, но сохранит вид (5), только вместо постоянных матриц вычетов $B_{i}$ будут стоять матрицы с полиномами от $1 /\left(z-a_{i}\right)$. Обозначим эти матричные функции через $\widetilde{B}_{i}$. Преобразование $\Gamma_{\alpha \beta}^{i}(z)$ при подходящем выборе постоянных $h_{\alpha \beta}^{i k}$ обнуляет элемент матрицы $\widetilde{B}_{i}$ системы $(3),(5)$, стоящий на месте $(\alpha-1, \beta)$, причем все остальные матрицы $\widetilde{B}_{j}$ сохраняют нижнетреугольность, а первые $\alpha-1$ строк системы вообще не изменятся. Элемент $e_{\alpha \beta}^{i}$ следует выбрать так: $e_{\alpha \beta}^{i}=a_{i} b_{\alpha-1, \beta}^{i}$, где $b_{\alpha-1, \beta}^{i}$ - элемент, стоящий на месте $(\alpha-1, \beta)$ в матрице $\tilde{B}_{i}$.

Так, действуя в нужном порядке, с помощью преобразования

$$
\Gamma(z)=\Gamma_{p, p-1}^{n-1} \cdots \Gamma_{p, p-1}^{1} \cdots * * * \cdots \Gamma_{3,1}^{n-1} \cdots \Gamma_{3,1}^{1} \Gamma_{2,1}^{n-1} \cdots \Gamma_{2,1}^{1}
$$


обнулим все элементы матрицы коэффициентов (5), кроме последней строки и кроме единичной наддиагонали вычета в нуле. Представим матрицу коэффициентов в виде

$$
\tilde{B}(z)=\frac{1}{z}\left(\begin{array}{cccc}
0 & 1 & 0 & 0 \\
\vdots & \ddots & \ddots & 0 \\
0 & \ldots & 0 & 1 \\
* & * & * & *
\end{array}\right)+\left(\begin{array}{cccc}
0 & \ldots & \ldots & 0 \\
\vdots & \vdots & \vdots & \vdots \\
0 & \ldots & \ldots & 0 \\
* & * & * & *
\end{array}\right) .
$$

K полученной системе применим калибровочное преобразование вида

$$
\tilde{y}=\Gamma_{f}(z) y, \quad \Gamma_{f}(z)=\left(\begin{array}{cccccc}
1 & 0 & \ldots & \ldots & \ldots & 0 \\
0 & 1 / z & 0 & \ldots & \ldots & 0 \\
\vdots & -1 / z^{2} & 1 / z^{2} & 0 & \ldots & 0 \\
\vdots & 2 / z^{3} & -2 / z^{3} & 1 / z^{3} & \ddots & \vdots \\
\vdots & \vdots & \vdots & \vdots & \ddots & 0 \\
0 & * & * & * & * & *
\end{array}\right) .
$$

Оно построено с помощью процедуры п. (А). Полученная после преобразования система преобразуется в уравнение стандартной заменой.

Это преобразование переводит исходную систему в систему (25). Теорема доказана.

ТЕОРема 10. По фуксову уравнению (1) можно построить стабильное расслоение с логарифмической связностью и типом расщепления $K=(n-2) D$ с теми же особъми точками и монодромией.

ДокАЗАТЕЛЬСТво. Пусть точки $a_{1}=0, a_{2}, \ldots, a_{n}$ - особые точки фуксова уравнения (1), а точка $z=\infty$ - неособая. По уравнению (1) с помощью замены

$$
y^{1}=u, \quad y^{2}=u^{\prime}, \quad \ldots, \quad y^{p}=u^{(p-1)}
$$

построим регулярную систему вида

$$
\frac{d y}{d z}=\left(\begin{array}{cccc}
0 & 1 & 0 & 0 \\
\vdots & \ddots & \ddots & 0 \\
0 & \cdots & 0 & 1 \\
-b_{p} & \cdots & \cdots & -b_{1}
\end{array}\right) y
$$

с теми же особыми точками и монодромией, что у уравнения (1). Легко видеть, что локально, в окрестности точки $z=a_{i}$, система (28) преобразованием вида

$$
\tilde{y}=\left(z-a_{i}\right)^{D} y
$$

приводится к фуксовой в точке $z=a_{i}$ системе. Преобразованная система будет иметь особенность в бесконечности. Чтобы преобразовать систему (28) к системе, фуксовой в точках $a_{2}, \ldots, a_{n}$ и не имеющей особенности в бесконечности, 
воспользуемся калибровочным преобразованием вида

$$
\tilde{y}=\Gamma_{\infty}(z) y, \quad \Gamma_{\infty}(z)=\prod_{i=2}^{n}\left(\frac{z-a_{i}}{z}\right)^{D}
$$

голоморфно обратимым в бесконечности.

Преобразованная система

$$
\frac{d \tilde{y}}{d z}=\widetilde{B}(z) \tilde{y}, \quad \widetilde{B}(z)=\Gamma_{\infty} B(z) \Gamma_{\infty}^{-1}+\frac{d \Gamma_{\infty}}{d z} \Gamma_{\infty}^{-1}
$$

фуксова в области $\overline{\mathbb{C}} \backslash\{0\}$. Система вида

$$
\frac{d \tilde{y}}{d z}=\widetilde{B}_{0}(z) \tilde{y}, \quad \widetilde{B}_{0}(z)=\Gamma_{0} B(z) \Gamma_{0}^{-1}+\frac{d \Gamma_{0}}{d z} \Gamma_{0}^{-1},
$$

с $\Gamma_{0}(z)=\prod_{i=2}^{n}\left(z-a_{i}\right)^{D}$ фуксова в нуле и связана с системой (29) следующим образом:

$$
\widetilde{B}(z)=z^{-(n-2) D} \widetilde{B}_{0} z^{(n-2) D}-(n-2) \frac{D}{z} .
$$

С помощью полученных систем на сфере Римана может быть построено голоморфное расслоение с логарифмической связностью. Определим его с помощью следующего координатного описания:

$$
(F, \nabla)=\left(U_{0} \ni 0, U_{\infty}=\overline{\mathbb{C}} \backslash\{0\}, \omega_{0}=\widetilde{B}_{0}(z), \omega_{\infty}=\widetilde{B}(z), g_{0 \infty}=z^{(n-2) D}\right) .
$$

Построенное расслоение имеет тип расщепления $K=(0,(n-2), \ldots,(n-2) \times$ $(p-1))$, как и требуется в условии теоремы. Остается показать, что построенное расслоение со связностью образуют стабильную пару. Докажем это.

Поскольку пара $(F, \nabla)$, построенная по неприводимому представлению монодромии (2), всегда стабильна, то нас интересует случай приводимой монодромии. Пусть представление монодромии связности $\nabla$ приводимо, это значит, что у пространства горизонтальных сечений $\nabla$ есть нетривиальное инвариантное подпространство. Отметим, что степень расслоения $F$ (подрасслоения $F_{1}$, соответствующего подпредставлению монодромии) равна сумме асимптотик горизонтальных сечений логарифмической связности (ограничения связности) по всем особым точкам. Соответственно, достаточно доказать, что

$$
\mu\left(F_{1}\right)=\frac{1}{l} \sum_{i=1}^{n} \sum_{j=1}^{l} \beta_{i}^{j}<\frac{1}{p} \sum_{i=1}^{n} \sum_{j=1}^{p} \beta_{i}^{j}=\mu(F),
$$

где $\beta_{i}^{1}, \ldots, \beta_{i}^{l}$ - показатели связности $\nabla$ в точке $z=a_{i}$, соответствующие $l$-мерному инвариантному подпространству пространства горизонтальных сечений $\nabla$, а показатели $\beta_{i}^{l+1}, \ldots, \beta_{i}^{p}$ дополняют их до всего набора показателей связности $\nabla$. Возможность такого дополнения доказана в [12].

Предположим, что у представления монодромии (2) связности $\nabla$ имеется подпредставление. Другими словами, пространство горизонтальных сечений 
(пространство решений соответствующей системы) имеет нетривиальное инвариантное подпространство. Здесь нам снова удобнее работать с системой вида (28). Рассмотрим базис $u_{1}(z), \ldots, u_{p}(z)$ пространства решений уравнения, причем такой, что первые $l$ его элементов образуют базис инвариантного подпространства. Заметим, что фундаментальная матрица $Y(z)$ системы $(28)$ имеет вид

$$
Y(z)=\left(\begin{array}{cccc}
u_{1}(z) & u_{2}(z) & \ldots & u_{p}(z) \\
u_{1}^{\prime}(z) & u_{2}^{\prime}(z) & \ldots & u_{p}^{\prime}(z) \\
\vdots & \vdots & \ddots & \vdots \\
u_{1}^{(p-1)}(z) & u_{2}^{(p-1)}(z) & \ldots & u_{p}^{(p-1)}(z)
\end{array}\right)
$$

Матрица $Y_{i}(z)=\left(z-a_{i}\right)^{D} Y(z)-$ фундаментальная матрица фуксовой в $z=a_{i}$ системы.

Построим по формуле (27) уравнение, фундаментальными решениями которого будут функции $u_{1}(z), \ldots, u_{l}(z)$, образующие инвариантное подпространство. Применение этой формулы корректно, так как функции $u_{1}(z), \ldots, u_{l}(z)$ линейно независимы и порождают подпространство, инвариантное относительно оператора монодромии. Полученное уравнение будет иметь особыми точками особые точки $a_{1}, \ldots, a_{n}$ исходного уравнения и еще точки $\left\{c_{i}\right\}$, где вронскиан $W\left(u_{1}, \ldots, u_{l}\right)$ обращается в нуль.

ЛЕмма 4. Построенное уравнение

$$
u^{(l)}+q_{1}(z) u^{(l-1)}+\cdots+q_{l}(z) u=0
$$

имеет лишь конечное число особенностей, или, другими словами, коэффициенты уравнения (31) суть мероморфные функции.

ДокАзАтельство. Достаточно показать, что множество нулей $\left\{c_{i}\right\}$ вронскиана $W\left(u_{1}, \ldots, u_{l}\right)$ не имеет предельных точек. Докажем это от противного. Действительно, пусть $c$ - предельная точка $\left\{c_{i}\right\}$. Рассмотрим два случая. Если $c \notin\left\{a_{1}, \ldots, a_{n}\right\}$, то голоморфная в $z=c$ функция $W\left(u_{1}(z), \ldots, u_{l}(z)\right)$ имеет бесконечно много нулей в любой окрестности точки $z=c$, тогда она тождественно равна нулю, но это противоречит линейной независимости функций $u_{1}(z), \ldots, u_{l}(z)$.

Теперь рассмотрим случай $c=a_{1}$ (выбор точки $a_{1}$ не ограничивает общности). В точке $z=a_{1}$ фундаментальная система решений уравнения (31) может быть представлена в виде (8):

$$
\left(u_{1}(z), \ldots, u_{l}(z)\right)=\left(v_{1}(z), \ldots, v_{l}(z)\right)\left(z-a_{1}\right)^{A_{1}}\left(z-a_{1}\right)^{E_{1}},
$$

где $v_{1}(z), \ldots, v_{l}(z)$ - голоморфные в $z=a_{1}$ функции, а матрицы $A_{1}$ и $E_{1}$ определены в (8). Заметим, что

$$
W\left(v_{1}(z), \ldots, v_{l}(z)\right)=W\left(u_{1}(z), \ldots, u_{l}(z)\right)\left(z-a_{1}\right)^{-\operatorname{tr} A_{1}}\left(z-a_{1}\right)^{-\operatorname{tr} E_{1}} .
$$

Так как функции $\left(z-a_{1}\right)^{A_{1}}$ и $\left(z-a_{1}\right)^{E_{1}}$ не обращаются в нуль вне точки $z=a_{1}$ (и еще точки $z=\infty$ ), а значит, в точках $\left\{c_{i}\right\}$, лежащих в достаточно малой окрестности $a_{1}$, в нуль обращается вронскиан $W\left(v_{1}(z), \ldots, v_{l}(z)\right)$, то 
они должны быть тождественно нулевыми, что противоречит условию, так как тогда и решения $u_{1}(z), \ldots, u_{l}(z)$ были бы линейно зависимыми. Теперь доказательство следует из компактности сферы Римана, на которой определено уравнение. Лемма доказана.

Таким образом, доказано, что особых точек уравнения (31), полученных из нулей вронскиана $W\left(u_{1}, \ldots, u_{l}\right)$, конечное число, и их можно обозначить через $c_{1}, \ldots, c_{s}$. Очевидно, что в этих точках решения имеют только нули, иначе у исходного уравнения (1) тоже была бы особенность. Это соображение пригодится нам в дальнейшем.

Построенное уравнение (31) регулярное, а значит, фуксово. Следовательно, функции

$$
r_{j}(z)=q_{j}(z)\left(\prod_{i=1}^{n}\left(z-a_{i}\right)^{j}\right)\left(\prod_{i=1}^{s}\left(z-c_{i}\right)^{j}\right)
$$

голоморфны в $\mathbb{C}$. Уравнение (31) можно использовать как соотношение, выражающее старшую производную любой из функций $u_{1}(z), \ldots, u_{l}(z)$ через младшие производные. С помощью этого соотношения нам удастся построить калибровочное преобразование, которое обнулит все элементы фундаментальной матрицы $Y_{0}(z)$, стоящие на пересечении первых $l$ столбцов и последних $p-l$ строк.

Уравнение и его производные дадут соотношения для функций $u_{k}^{(l)}(z), \ldots$, $u_{k}^{(p-1)}(z)$ при $k=1, \ldots, l$. Легко проверить, что соотношения будут иметь вид

$$
u^{(m)}={ }^{m} \tilde{q}_{1}(z) u^{(m-1)}+\cdots+{ }^{m} \tilde{q}_{m}(z) u, \quad m \geqslant l,
$$

где функции

$$
\tilde{r}_{j}(z)=\tilde{q}_{j}(z) \prod_{i=1}^{n}\left(z-a_{i}\right)^{j}
$$

тоже голоморфны в $\mathbb{C}$, так как все уравнения (32) фуксовы. Соотношения (32) для $u^{(m)}(z)$ получены из уравнения $(31)$ и $p-l-1$ его производных, которые, очевидно, тоже являются фуксовыми уравнениями.

Применим к фундаментальной матрице системы (28) калибровочное преобразование

$$
\tilde{y}=\Gamma(z) y
$$

где

$$
\Gamma(z)=\left(\begin{array}{cccccc}
1 & & & & & \\
0 & \ddots & & & 0 & \\
0 & 0 & 1 & & & \\
-{ }^{l} \tilde{q}_{l}(z) & \ldots & -{ }^{l} \tilde{q}_{1}(z) & 1 & & \\
\vdots & & & \ddots & \ddots & \\
-{ }^{p-1} \tilde{q}_{p-1}(z) & \ldots & \ldots & \ldots & -{ }^{p-1} \tilde{q}_{1}(z) & 1
\end{array}\right)
$$

(преобразование (33) для всех $m=l, \ldots, p-1$ вычитает из строки с номером $m+1$ предыдущие $m$ строк, обнуляя при этом первые $l$ элементов $(m+1)$-й строки матрицы $Y(z))$. 
Матрицы

$$
\begin{aligned}
\widetilde{\Gamma}_{0}(z) & =\Gamma_{0}(z) \Gamma(z) \Gamma_{0}^{-1}(z), \\
\widetilde{\Gamma}_{\infty}(z) & =\Gamma_{\infty}(z) \Gamma(z) \Gamma_{\infty}^{-1}(z)
\end{aligned}
$$

по построению голоморфно обратимы в областях

$$
U_{0} \backslash\left\{c_{1}, \ldots, c_{s}\right\}, \quad U_{\infty} \backslash\left\{c_{1}, \ldots, c_{s}\right\}
$$

соответственно, поэтому преобразования $\tilde{y}=\widetilde{\Gamma}_{0}(z) y, \tilde{y}=\widetilde{\Gamma}_{\infty}(z) y$, примененные к системам (30), (29) соответственно, переводят матричные дифференциальные 1-формы $\omega_{0}, \omega_{\infty}$, задающие мероморфную связность $\nabla$ с особенностями $a_{1}, \ldots, a_{n}$ в расслоении $F$, в матричные дифференциальные 1-формы

$$
\begin{aligned}
\tilde{\omega}_{0} & =\widetilde{\Gamma}_{0} \omega_{0} \widetilde{\Gamma}_{0}^{-1}+\left(d \widetilde{\Gamma}_{0}\right) \widetilde{\Gamma}_{0}^{-1}, \\
\tilde{\omega}_{\infty} & =\widetilde{\Gamma}_{\infty} \omega_{\infty} \widetilde{\Gamma}_{\infty}^{-1}+\left(d \widetilde{\Gamma}_{\infty}\right) \widetilde{\Gamma}_{\infty}^{-1}
\end{aligned}
$$

блочно-верхнетреугольного вида, задающие мероморфную связность $\widetilde{\nabla}$ с особенностями $a_{1}, \ldots, a_{n}, c_{1}, \ldots, c_{s}$ в том же расслоении $F\left(\right.$ хотя $\widetilde{\Gamma}_{0}(z), \widetilde{\Gamma}_{\infty}(z)$ и имеют особенности в $U_{0}, U_{\infty}$ соответственно):

$$
\tilde{g}_{0 \infty}=\widetilde{\Gamma}_{0} g_{0 \infty} \widetilde{\Gamma}_{\infty}^{-1}=\left(\Gamma_{0} \Gamma \Gamma_{0}^{-1}\right) \Gamma_{0} \Gamma_{\infty}^{-1}\left(\Gamma_{\infty} \Gamma^{-1} \Gamma_{\infty}^{-1}\right)=\Gamma_{0} \Gamma_{\infty}^{-1}=g_{0 \infty} .
$$

Таким образом, расслоение $F$ имеет подрасслоение $\widetilde{F}^{1} \subset F$, которое стабилизируется связностью $\widetilde{\nabla}$, и

$$
\widetilde{F}^{1} \cong \mathscr{O}(0) \oplus \mathscr{O}(n-2) \oplus \cdots \oplus \mathscr{O}((l-1)(n-2)) .
$$

Следовательно,

$$
\mu\left(\widetilde{F}^{1}\right)=(n-2) \frac{l-1}{2}<(n-2) \frac{p-1}{2}=\mu(F) .
$$

С другой стороны, обозначив через $\widetilde{\nabla}^{1}, \nabla^{1}$ ограничения связностей $\widetilde{\nabla}, \nabla$ на подрасслоения $\widetilde{F}^{1}, F^{1}$ соответственно, будем иметь:

$$
\begin{aligned}
\operatorname{deg} \widetilde{F}^{1} & =\sum_{i=1}^{n} \operatorname{tr} \operatorname{res}_{a_{i}} \widetilde{\nabla}^{1}+\sum_{k=1}^{h} \operatorname{tr} \operatorname{res}_{c_{k}} \widetilde{\nabla}^{1} \\
& =\sum_{i=1}^{n} \operatorname{tr} \operatorname{res}_{a_{i}} \nabla^{1}+\sum_{k=1}^{h} \operatorname{res}_{c_{k}} \frac{d W\left(u_{1}, \ldots, u_{l}\right)}{W\left(u_{1}, \ldots, u_{l}\right)}=\operatorname{deg} F^{1}+H
\end{aligned}
$$

где

$$
H=\sum_{k=1}^{h} \operatorname{res}_{c_{k}} \frac{d W\left(u_{1}, \ldots, u_{l}\right)}{W\left(u_{1}, \ldots, u_{l}\right)}=\sum_{k=1}^{h} \operatorname{ord}_{c_{k}} W\left(u_{1}, \ldots, u_{l}\right) \geqslant 0
$$

(равенства $\operatorname{tr} \operatorname{res}_{a_{i}} \widetilde{\nabla}^{1}=\operatorname{trres}_{a_{i}} \nabla^{1}$ следуют из голоморфной эквивалентности в точках $a_{i}$ соответствующих матричных дифференциальных 1-форм, задающих связности $\widetilde{\nabla}^{1}$ и $\nabla^{1}$ ).

Итак,

$$
\mu\left(F^{1}\right) \leqslant \mu\left(\widetilde{F}^{1}\right)<\mu(F),
$$

что доказывает стабильность пары $(F, \nabla)$. Теорема 10 доказана. 


\section{4. Некоторые следствия}

\section{1. Контрпримеры к проблеме Римана-Гильберта для скалярных} фуксовых уравнений. Введем два класса представлений. Б-представлениями называют приводимые представления, каждая образующая которых приводится к жордановой клетке. В этом классе А. А. Болибрухом были найдены первые контрпримеры к проблеме Римана-Гильберта для фуксовых систем. Еще один класс представлений - это представления вида $\chi=\chi_{1} \oplus \chi_{2}$ с образующими $G_{i}=G_{i}^{1} \oplus G_{i}^{2}, i=1, \ldots, n$, такими, что спектры матриц $G_{i}^{1}$ и $G_{i}^{2}$ не пересекаются ни при одном $i$. Назовем их расщепимыми.

СлЕДСТвИЕ 2. Б-представления и расщепимые представления с не менее чем тремя образующими не могут быть реализованы как представления монодромии скалярных фуксовых уравнений.

ДокАЗАТЕЛЬСтво. Для обоих классов представлений можно показать, что по ним нельзя построить стабильную пару. Для Б-представлений это легко следует из доказательства теоремы 11.2 из [3].

Если представление расщепимо, $\chi=\chi_{1} \oplus \chi_{2}$, то построенное по нему расслоение $F$ со связностью имеет два подрасслоения $F_{1}$ и $F_{2}$, которые стабилизируются связностью (инвариантны относительно действия монодромии). Подпредставлениям $\chi_{1}$ и $\chi_{2}$ отвечают подрасслоения $F_{1}$ и $F_{2}$ соответственно. Покажем, что

$$
\operatorname{deg} F=\operatorname{deg} F_{1}+\operatorname{deg} F_{2} .
$$

В качестве ассоциированного базиса рассмотрим слабо левелевский. Инвариантные подпространства, отвечающие прямым слагаемым монодромии, порождаются частями слабо левелевского базиса, т. е. первые $l$ векторов порождают первое инвариантное подпространство, а последние $p-l$ векторов - второе. Действительно, рассмотрим оператор $G_{i}=G_{i}^{\prime} \oplus G_{i}^{\prime \prime}$, где $G_{1}^{\prime}$ и $G_{i}^{\prime \prime}$ - образующие подпредставлений. По условию, операторы $G_{i}^{\prime}$ и $G_{i}^{\prime \prime}$ не имеют одинаковых собственных значений, а значит, каждый вектор из слабо левелевского базиса лежит ровно в одном из подпространств - в том, в котором лежит соответствующее собственное подпространство. Тогда степень подрасслоения $F_{1}$ равна сумме показателей, соответствующих подпредставлению $\chi_{1}$ :

$$
\operatorname{deg} F_{1}=\sum_{i=1}^{n} \sum_{j=1}^{l} \beta_{i}^{j},
$$

то же верно и для $F_{2}$, т. е.

$$
\operatorname{deg} F_{2}=\sum_{i=1}^{n} \sum_{j=l+1}^{p} \beta_{i}^{j} .
$$

Следовательно, выполнено (35). Из (35) следует, что пара $(F, \nabla)$ не может быть стабильной (аналогичное рассуждение см. в [13]).

Таким образом, из третьей формулировки 21-й проблемы Гильберта следует, что для этих двух классов представлений она имеет отрицательное решение. Следствие доказано. 
ЗАмечАниЕ 1. В хорошо изученном классе фуксовых уравнений второго порядка с тремя особыми точками 0,1 и $\infty$ указанные семейства представлений дают все классы, не реализуемые такими уравнениями. В частности, к этому классу относится гипергеометрическое уравнение.

Это нетрудно показать, воспользовавшись полученными формулировками. Например, по неприводимому представлению можно построить фуксову систему, а она постоянной калибровкой обязана приводиться к виду (3), (5). Действительно, вычет в точке $z=1$ матрицы коэффициентов можно привести к нижнетреугольному виду, а вычет в нуле не сможет иметь такой вид, так как система неприводима. Далее, подходящим диагональным калибровочным преобразованием сделаем правый верхний элемент вычета в нуле единицей. Еще более удобной является третья формулировка, но она требует применения техники работы с расслоениями со связностью.

СлЕДСТВИЕ 3. По монодромии фуксова уравнения можно построить бесконечное семейство фуксовых систем.

ДокАзАтельство. Согласно теореме 10, по фуксову уравнению можно построить стабильную пару $(F, \nabla)$. С другой стороны, из теоремы 3 работы [14] вытекает, что существование стабильной пары гарантирует существование искомого семейства систем. Следствие доказано.

Основная трудность явного предъявления контрпримеров к 21-й проблеме Гильберта для скалярных фуксовых уравнений состоит в сложной зависимости от положения особых точек. Как будет показано ниже (см. следствие 4), в случае $n \geqslant 4$ особых точек ответ к проблеме неустойчив относительно движения особых точек. Известным примером такой зависимости является случай четырех особых точек $0,1, t$ и $\infty$ и неприводимой монодромии, принадлежащей $\mathrm{SL}(2, \mathbb{C})$. Такие данные, как правило, нельзя реализовать скалярным фуксовым уравнением с четырьмя особыми точками, но можно реализовать уравнением с одной дополнительной, так называемой “ложной” (без ветвления), особой точкой $w(t)$, положение которой зависит от $t$. Мы предполагаем, что монодромия постоянна. Функция $w(t)$ является решением шестого уравнения Пенлеве (см. [15]). Как правило, решения уравнений Пенлеве - это новые неизвестные функции, имеющие кроме точек $0,1, \infty$ еще и другие, так называемые подвижные, особенности. Эти особенности - те положения точки $t$, при которых существует скалярное фуксово уравнение, реализующее заданную монодромию. Таким образом, вопрос о возможности построения скалярного фуксова уравнения - это вопрос описания структуры дивизора.

4.2. Недеформируемость фуксовых уравнений. Известно, что фуксову систему с набором особых точек $a=\left(a_{1}, \ldots, a_{n}\right)$ можно включить в семейство фуксовых систем

$$
\frac{d y}{d z}=\left(\sum_{i=1}^{n} \frac{B_{i}(a)}{z-a_{i}}\right) y, \quad \sum_{i=1}^{n} B_{i}(a)=0,
$$

зависящее от параметра $a$ так, что эти системы будут иметь одно и то же представление монодромии (2). Такое семейство систем называется изомо- 
нодромным. Наиболее известное из таких семейств, задаваемое уравнением Шлезингера, называется шлезингеровским. В нерезонансном случае все изомонодромные деформации шлезингеровские или сводятся к ним сопряжением. Непрерывные изомонодромные деформации сохраняют показатели. Шлезингеровская деформация отличается от других еще и тем, что при ней сохраняются связи (отношения $Y_{i} / Y_{j}$ ) между ассоциированными базисами в разных точках. Аналогично деформациям систем можно определить деформацию расслоений со связностью.

В этом пункте мы будем активно применять технику работы [16].

Теорема 11. Стабильная пара $(F(a), \nabla(a))$ с типом расщепления $K=$ $\left(k_{1}, \ldots, k_{p}\right)$, где $k_{l+1}-k_{l}>1$ для некоторого $l$, при непрерывной деформачии по параметру а не может сохранить тип расщепления.

ДокАзАТЕЛЬСтво. В работе [16] показано, что по паре $(F(a), \nabla(a))$ можно построить фуксову систему с той же монодромией, что у связности $\nabla$, и с особыми точками $a_{1}, \ldots, a_{n}, \infty$ :

$$
\frac{d y}{d z}=\left(\sum_{i=1}^{n} \frac{{ }^{*} B_{i}(a)}{z-a_{i}}\right) y, \quad \sum_{i=1}^{n}{ }^{*} B_{i}=-K,
$$

где $K$ - тип расщепления расслоения $F$. Тогда, согласно теореме Левеля, фундаментальная матрица, соответствующая ассоциированному базису в бесконечности, примет вид

$$
Y(z)=U(z, a) z^{K}, \quad U(z)=I+U_{1}(a) \frac{1}{z}+\cdots .
$$

То, что $K$ - не только набор нормирований в бесконечности, но и тип расщепления, означает, что

$$
Y(z)=U(z, a) z^{K}=z^{K} V(z, a), \quad V(z)=I+V_{1}(a) \frac{1}{z}+\cdots .
$$

Таким образом,

$$
U(z, a)=I+U_{1}(a) z^{-1}+O\left(z^{-2}\right)=z^{K} V(z, a) z^{-K}
$$

Отсюда, а также из того, что $k_{i+1}-k_{i}>1$, следует, что

$$
u_{i j}(a) \equiv 0, \quad i \leqslant l<j .
$$

С другой стороны, из формулы

$$
\frac{\partial U_{1}(a)}{\partial a_{i}}={ }^{*} B_{i}(a)
$$

полученной в [16] из уравнения Шлезингера и пфаффовой формы, задающей шлезингеровскую изомонодромную деформацию, следует, что система (36) имеет блочно-нижнетреугольную форму. Но это, в свою очередь, означает, 
что подсистема, полученная в пересечении последних $l$ столбцов и строк, задает логарифмическую связность в подрасслоении $F_{1}$ с типом расщепления $\left(k_{p-l+1}, \ldots, k_{p}\right)$, что противоречит стабильности пары $(F, \nabla)$ :

$$
\mu\left(F_{1}\right)=\frac{k_{p-l+1}+\cdots+k_{p}}{l}>\frac{k_{1}+\cdots+k_{p}}{p}=\mu(F) .
$$

Теорема доказана.

Множество наборов $a=\left(a_{1}, \ldots, a_{n}\right)$ определяется равенством нулю некоторых элементов матрицы коэффициентов вспомогательной системы, соответственно, оно является аналитическим подмножеством $\mathbb{C}^{n}$ положительной коразмерности.

Назовем уравнение резонансным, если какие-то его показатели (в одной точке) отличаются на целое число.

СлЕДСТвИЕ 4. Нерезонансное уравнение $c$ набором особых точек $a=$ $\left(a_{1}, \ldots, a_{n}\right), n \geqslant 4$, и монодромией $\chi$ не может быть включено в изомонодромное семейство с параметрами $a \in \Omega$, где $\Omega$ - некоторая область в $\mathbb{C}^{n}$.

ДокАзАтельство. Согласно третьей формулировке, существование уравнения эквивалентно существованию стабильной пары $(F, \nabla)$ с типом расщепления $K=(0,(n-2), \ldots,(p-1)(n-2))$. По условию $n-2>1$, соответственно, это расслоение подпадает под условие теоремы 11 , которая утверждает, что не существует изомонодромной деформации шлезингеровского типа, сохраняющей тип расщепления. Все деформации в нерезонансном случае сводятся к шлезингеровским, следовательно, их не существует. Следствие доказано.

Здесь также можно сказать, что множество наборов $a=\left(a_{1}, \ldots, a_{n}\right)$, при которых существует фуксово уравнение с заданной монодромией, является аналитическим множеством положительной коразмерности. В резонансном случае эти утверждения будут верными, но только для деформаций, сохраняющих связи между локальными системами.

В заключение я хочу выразить глубокую благодарность Д. Бертрану, обратившему мое внимание на эти вопросы, и Р. Р. Гонцову, чьи замечания позволили значительно улучшить эту работу.

\section{Список литературы}

[1] Б. Риман, Сочинения, Гостехиздат, М., 1948, 543 с.; англ. изд.: В. Riemann, Collected papers, Translated from the 1892 German edition, Kendrick Press, Heber City, UT, 2004, ISBN: 0-9740427-2-2; 0-9740427-3-0, x+555 pp.

[2] Д. Гильберт, Избранные труды, 2, М., Факториал, 1998, ISBN: 5-88688-028-3, $607 \mathrm{c}$.

[3] А.А. Болибрух, Обратные задачи монодромии в аналитической теории дифференциальных уравнений, МЦНМО, М., 2009, ISBN: 978-5-94057-510-8, 220 с., http://biblio.mccme.ru/node/2154.

[4] А. А. Болибрух, "Проблема Римана-Гильберта на комплексной проективной прямой", Матем. заметки, 46:3 (1989), 118-120. 
[5] А. А. Болибрух, "21-я проблема Гильберта для линейных фуксовых систем”, Тр. МИАН, 206, Наука, М., 1994, 3-158; англ. пер.: А. A. Bolibrukh, The 21st Hilbert problem for linear Fuchsian systems, Proc. Steklov Inst. Math., 206, Amer. Math. Soc., Providence, RI, 1995, viii, 145 pp.

[6] И.В. Вьюгин, Р. Р. Гонцов, "О дополнительных параметрах в обратных задачах монодромии", Матем. сб., 197:12 (2006), 43-64; англ. пер.: I. V. V'yugin, R. R. Gontsov, "Additional parameters in inverse monodromy problems", Sb. Math., 197:12 (2006), 1753-1773.

[7] M. van der Put, M. Singer, Galois theory of linear differential equations, Grundlehren Math. Wiss., 328, Springer-Verlag, Berlin, 2003, ISBN: 3-540-44228-6, xviii+438 pp.

[8] И. В. Вьюгин, "Построение фуксовой системы по фуксову уравнению", Вестник КГПИ: Математические и естественные науки, 2:3 (2007), 43-47.

[9] И. В. Вьюгин, Р. Р. Гонцов, "О построении системы линейных дифференциальных уравнений по скалярному уравнению”, Тр. МИАН, Наука, М., 2010 (в печати).

[10] И. В. Вьюгин, "О 21-й проблеме Гильберта для скалярных фуксовых уравнений”, Докл. РАН, 425:3 (2009), 305-308; англ. пер.: V. I. V'yugin, "Hilbert's 21st problem for scalar Fuchsian equations", Dokl. Math., 79:2 (2009), 203-206.

[11] P. Deligne, "Équations différentielles à points singuliers réguliers", Lecture Notes in Math., 163 (1970), 133 pp.

[12] A. I. Gladyshev, "On the Riemann-Hilbert problem in dimension 4", J. Dyn. Control Syst., 6:2 (2000), 219-264.

[13] И.В. Вьюгин, "Фуксовы системы с вполне приводимой монодромией", Матем. заметки, 85:6 (2009), 817-825; англ. пер.: I. V. V'yugin, "Fuchsian systems with completely reducible monodromy", Math. Notes, 85:6 (2009), 780-786.

[14] А. А. Болибрух, "Проблема Римана-Гильберта на компактной римановой поверхности", Монодромия в задачах алгебрачческой геометрии и дифференииальных уравнений, Сб. статей, Тр. МИАН, 238, Наука, М., 2002, 55-69; англ. пер.: A. A. Bolibrukh, "The Riemann-Hilbert problem on a compact Riemann surface", Proc. Steklov Inst. Math., 238 (2002), 47-60.

[15] Р. Р. Гонцов, В. А. Побережный, "Различные варианты проблемы Римана-Гильберта для линейных дифференциальных уравнений”, УМН, 63:4 (2008), 3-42; англ. пер.: R. R. Gontsov, V. A. Poberezhnyi, "Various versions of the Riemann-Hilbert problem for linear differential equations", Russian Math. Surveys, 63:4 (2008), 603-639.

[16] А. А. Болибрух, "O tau-функции уравнения изомонодромных деформаций Шлезингера", Матем. заметки, 74:2 (2003), 184-191; англ. пер.: A. A. Bolibrukh, "On the tau function for the Schlesinger equation of isomonodromic deformations", Math. Notes, 74:2 (2003), 177-184.

И. В. Вьюгин (I. V. Vyugin)

Поступила в редакцию

Институт проблем передачи информации

08.12.2010 им. А. А. Харкевича РАН

E-mail: vyugin@gmail.com 